\title{
Role of Nicotinic Acetylcholine Receptor a3 and a7 Subunits in Detrusor Overactivity Induced by Partial Bladder Outlet Obstruction in Rats
}

\author{
Hyo Sin Kim, Wang Jin Park, Eun Young Park, Jun Sung Koh, Tae-Kon Hwang, Joon Chul Kim \\ Department of Urology, The Catholic University of Korea College of Medicine, Seoul, Korea
}

Purpose: To investigate the role of $\alpha 3$ and $\alpha 7$ nicotinic acetylcholine receptor subunits (nAChRs) in the bladder, using a rat model with detrusor overactivity induced by partial bladder outlet obstruction (BOO).

Methods: Forty Sprague-Dawley rats were used: 10 were sham-operated (control group) and 30 were observed for 3 weeks after partial BOO. BOO-induced rats were further divided into 3 groups: Two groups of 10 rats each received intravesicular infusions with hexamethonium (HM group; $n=10$ ) or methyllycaconitine (MLC group; $n=10$ ), which are antagonists for $\alpha 3$ and $\alpha 7 \mathrm{nAChRs,} \mathrm{respectively.} \mathrm{The} \mathrm{remaining} \mathrm{BOO-induced} \mathrm{rats} \mathrm{received} \mathrm{only} \mathrm{saline} \mathrm{infusion} \mathrm{(BOO} \mathrm{group;} \mathrm{n}=10$ ). Based on the contraction interval measurements using cystometrogram, the contraction pressure and nonvoiding bladder contractions were compared between the control and the three BOO-induced groups. Immunofluorescent staining and Western blotting were used to analyze $\alpha 3$ and $\alpha 7$ nAChRs levels.

Results: The contraction interval of the MLC group was higher than that of the BOO group $(\mathrm{P}<0.05)$. Nonvoiding bladder contraction almost disappeared in the HM and MLC groups. Contraction pressure increased in the BOO group $(\mathrm{P}<0.05)$ compared with the control group and decreased in the HM and MLC groups compared with the BOO group $(\mathrm{P}<0.05)$. Immunofluorescence staining showed that the $\alpha 3 \mathrm{nAChR}$ signals increased in the urothelium, and the $\alpha 7 \mathrm{nAChR}$ signals increased in the urothelium and detrusor muscle of the BOO group compared with the control group. Western blot analysis showed that both $\alpha 3$ and $\alpha 7 \mathrm{nAChR}$ levels increased in the BOO group $(\mathrm{P}<0.05)$.

Conclusions: Alpha3 and $\alpha 7 \mathrm{nAChRs}$ are associated with detrusor overactivity induced by BOO. Furthermore, nAChR antagonists could help in clinically improving detrusor overactivity.

Keywords: Urinary Bladder, Overactive; Alpha7 Nicotinic Acetylcholine Receptor; Urinary Bladder Neck Obstruction

- Research Ethics: Animals were handled in accordance with the National Institutes of Health Guide for the Care and Use of Laboratory Animals, and experimental protocols were approved by the Institutional Animal Care (CUMC-2008-0012-02) and Use Committee of the Catholic University of Korea.

- Conflict of Interest: No potential conflict of interest relevant to this article was reported.

\section{INTRODUCTION}

Acetylcholine (Ach) is one of the major regulators of urinary bladder function and is mainly released from the postganglion- ic fiber terminals to induce contraction [1]. However, a nonneuronal release of Ach has been shown in the bladder, bronchial, and corneal epithelia and digestive tract mucosa. These nonneurogenic Ach molecules act in an autocrine or paracrine

Corresponding author: Joon Chul Kim (iD) http://orcid.org/0000-0002-4019-620X Department of Urology, Bucheon St. Mary's Hospital, The Catholic University of Korea College of Medicine, 327 Sosa-ro 327beon-gil, Wonmi-gu, Bucheon 420-717, Korea

E-mail: kjc@catholic.ac.kr / Tel: +82-32-340-7071 / Fax: +82-32-340-2124 Submitted: February 26, 2015 / Accepted after revision: March 10, 2015 
manner [2,3]. Similarly, urothelium also secretes neurotransmitters in response to extrinsic stimulation when the bladder is filled and distended; Ach is released particularly in the bladder filling phase [4,5]. According to several studies, the urothelium acts primarily as a barrier to urine but also expresses various types of receptors and responds actively to extrinsic stimulation, thus helping to regulate bladder function $[6,7]$.

Several studies have been conducted to examine the muscarinic and purinergic receptors in the urothelial layer of the bladder. It has been postulated that the nicotinic Ach receptor (nAChR) might be associated with the induction of overactive bladder (OAB) symptoms [8,9]. nAChR regulates bladder function by mediating fast synaptic transmission and is also involved in the contraction and relaxation of the bladder in the autonomic nervous system [5]. To date, 17 types of nAChR have been reported, including 10 alpha ( $\alpha$ ) subtypes and 4 beta $(\beta)$ subtypes. In addition, there are gamma $(\gamma)$, delta $(\delta)$, and epsilon ( $\varepsilon$ ) subtypes [10-14]. Beckel et al. [15] reported that the $\alpha 3$ and $\alpha 7$ type nAChRs in the urothelium of rats were involved in regulating bladder function, and the inhibition of $\alpha 3$ receptors resulted in an inhibition of reflex voiding, but the inhibition of $\alpha 7$ receptors reduced the inhibition of reflex voiding.

Bladder outlet obstruction (BOO) is one of the causes for $\mathrm{OAB}$. OAB arises from the functional, pharmacological, and molecular biological changes following the onset of BOO. Of these, changes in various types of receptors present in the bladder have been of increasing interest, and to date, studies have focused on muscarinic receptors [16]. However, little is known about the role of nAChRs.

Therefore, we attempted to examine the expression of $a 3$ and a7 nAChRs in the bladder. We used rats with detrusor overactivity induced by partial BOO. We also investigated the changes in the functions of the bladder corresponding to the subunits of nAChR following the administration of specific antagonists of the $\alpha 3$ and $\alpha 7$ nAChRs.

\section{MATERIALS AND METHODS}

\section{Experimental Animals}

We used 40 male Sprague-Dawley rats, weighing 450 to $500 \mathrm{~g}$ (16 weeks old). They were divided into control group $(n=10)$ and BOO-induced group $(\mathrm{n}=30)$. For the control group, a sham operation was performed. In the BOO-induced group, a partial BOO was attempted. Animals were handled in accordance with the National Institutes of Health Guide for the Care and Use of Laboratory Animals, and experimental protocols were approved by the Institutional Animal Care (CUMC-20080012-02) and Use Committee of the Catholic University of Korea.

\section{Partial BOO}

Experimental animals were anesthetized using an intramuscular injection of 15-mg/kg ketamine (Yuhan Co., Seoul, Korea) and 5-mg/kg xylazine (Rompun, Bayer Korea Co., Seoul, Korea). With the rats in the supine position, a longitudinal incision was made in the lower abdomen. The bladder neck and periurethral area were dissected out. A 25-G needle sheath was inserted into the urethra and the bladder neck was ligated using a 3-0 silk, after which the sheath was removed. In the sham-operated control animals, the bladder neck was very loosely ligated to not induce any obstruction.

\section{Assessment of Bladder Functions}

Three weeks following the obstruction, cystometrogram was performed in the control and BOO-induced groups. Of the 30 rats with $\mathrm{BOO}, 10$ were given an intravesicular administration of the $\alpha 3 \mathrm{nAChR}$ antagonist, hexamethonium (HM group); 10 were administered the $\alpha 7 \mathrm{nAChR}$ antagonist, methyllycaconitine citrate (MLC group), and 10 were received only saline infusion (BOO group).

A suprapubic midline incision was made under urethane anesthesia (12 mg/kg) and the bladder was identified. A 25-G needle connected to polyethylene tubing was placed in the bladder dome. The tubing was connected to a pressure transducer and an infusion pump using a 3-way stopcock. The bladder was emptied and saline was infused into the bladder at 0.04 $\mathrm{mL} / \mathrm{min}$ using a Harvard syringe pump. Intravesical pressure was recorded using a polygraph apparatus (Grass 7D, Grass Instrument Co., Quincy, MA, USA); the contraction interval, contraction pressure, and presence of nonvoiding contraction (NVC) were examined. NVCs were defined as the rise in intravesical pressure exceeding $4 \mathrm{cmH}_{2} \mathrm{O}$ during the filling phase but not during voiding of urine. The Harvard syringe pump was stopped in the HM and MLC rats and the bladder was emptied for the administration of the nAChR antagonists. Voiding activity was prevented by clamping the penis. Saline infusions $(0.2 \mathrm{~mL})$ containing $80 \mathrm{mM} \mathrm{HM}$ or $1 \mathrm{mM} \mathrm{MLC}$ were instilled using a $25-\mathrm{G}$ needle placed superior to the bladder. One hour later, saline was infused into the bladder at $0.04 \mathrm{~mL} /$ min using a Harvard syringe pump. The BOO group also re- 
ceived the same treatment without the drugs for 1-hour bladder retention and the intravesicular pressure was recorded.

\section{Tissue Extraction}

After the bladder function test, the bladder and urethra of the control and BOO-induced groups were dissected from the adjacent tissue. The bladder was cut above the trigone region and was used for immunofluorescent staining and Western blot analysis.

\section{Immunofluorescent Staining of $\alpha 3$ and $a 7$ nAChRs}

Thawed tissue samples (stored in liquid nitrogen) were fixed in $4 \%$ paraformaldehyde and placed in $0.5 \mathrm{M}$ sucrose at $4^{\circ} \mathrm{C}$. Using the optimal cutting temperature solution, polyethylene glycol, the tissues were sectioned at $3 \mu \mathrm{m}$. The tissue sections were then rinsed with phosphate buffered saline (PBS; pH 7.4). To inhibit nonspecific immunofluorescence staining, slides were exposed to a blocking solution (1.5\% normal goat serum, $1.5 \%$ normal horse serum, $1 \%$ BSA, $0.1 \%$ Triton X-100 in PBS) at room temperature for 1 hour. Antibodies to a $3 \mathrm{nAChR}$ (sc1771; 1:200; Santa Cruz Biotechnology Inc., Santa Cruz, CA, USA) and $\alpha 7$ nAChR (sc-5544; 1:200; Santa Cruz Biotechnology Inc.) were then applied and incubated for 2 hours at room temperature. The slide was rinsed with PBS three times and then incubated in the secondary antibody, Alexa Fluor 488 labeled goat antirabbit IgG (1:300; Molecular Probes, Eugene, OR, USA) at room temperature for 1 hour. The sample was rinsed with PBS three times. Slides were stained with 4',6-diamidino-2-phenylindole (Vector Laboratories, Burlingame, CA, USA), and mounted for examination using a light microscope (Axiovert 200, Zeiss, Oberkochen, Germany). The immunoreactivity was expressed as mean \pm standard error of the mean (SEM) using a scoring system according to the degree of staining ( 5 , very strong; 4 , strong; 3 , intermediate; 2 , weak; 1 , very weak). Thus, the distribution and intensity of $\alpha 3$ and $\alpha 7$ nAChRs were measured by 1 urologist and 2 pathologists.

\section{Western Blotting}

The tissue was ground in liquid nitrogen and processed using a Qproteome Mammalian Protein Prep Kit (Qiagen, Hilden, Germany). The prepared sample was centrifuged at $4^{\circ} \mathrm{C}$ and $14,000 \mathrm{rpm}$ for 10 minutes. Total protein was measured using a Bradford dye-binding protein assay kit (Bio-Rad Laboratories Inc., Hertfordshire, CA, USA) according to the manufacturer's instructions.
Protein $(30 \mu \mathrm{g})$ and $2 \times$ sample buffer (Bio-Rad Laboratories Inc.) were mixed at a ratio of 1:1. The reaction was performed at $100^{\circ} \mathrm{C}$ for 5 minutes and then stopped at $4^{\circ} \mathrm{C}$. Electrophoresis was performed at $4^{\circ} \mathrm{C}$ and $70 \mathrm{~V}$ for 2 hours, using a Mini-PROTEAN three-cell unit (Bio-Rad Laboratories Inc.). Isolated proteins were transferred to a polyvinylidene difluoride membrane (Millipore Corporation, Bedford, MA, USA) using a transblot SD semidry system (Bio-Rad Laboratories Inc.) based on six gels at $4^{\circ} \mathrm{C}$ at a voltage of $20 \mathrm{~V}$ for 90 minutes. The membrane was blocked using 10\% skim milk. Primary antibodies to a 3 nAChR (57 KDa: ab65181, Abcam PLC, Cambridge, UK), a7 nAChR (56 KDa: ab24644, Abcam PLC), and glyceraldehyde 3-phosphate dehydrogenase (36 KDa: ab37168, Abcam PLC) were diluted 1:500 using 5\% skim milk. The reaction was run at room temperature for 1 hour. After the membrane was rinsed, enzyme-conjugated secondary antibodies, diluted in 5\% skim milk at a ratio of 1:10,000, to $\alpha 3 \mathrm{nAChR}$ and glyceraldehyde 3-phosphate dehydrogenase were reacted with rabbit IgG-HRP (ab6721: Abcam PLC) and that of $a 7 \mathrm{nAChR}$ was reacted with rat IgG-HRP (ab6734: Abcam PLC) at room temperature for 30 minutes. After the membrane was rinsed, the reaction using enhanced chemiluminescence solution (Amersham Biosciences, Buckinghamshire, UK) was performed for 1 minute. The membrane was placed on a photographic film and developed. Using a densitometer (LAS-3000, Fuji Film, Tokyo, Japan), quantitative analyses of $\alpha 3$ and $\alpha 7 \mathrm{nAChRs}$ levels were performed for the urothelium and detrusor muscle in the control and $\mathrm{BOO}$ groups.

\section{Statistical Analysis}

All data were analyzed using SPSS ver. 17.0 (SPSS Inc., Chicago, IL, USA). The data were expressed as mean \pm SEM. For comparisons among the groups, one-way analysis of variance and Newman-Keuls multiple comparison test were performed with $\mathrm{P}<0.05$ as an indication of statistical significance.

\section{RESULTS}

\section{Changes in the Contraction Interval and Contraction Pressure of the Bladder in Cystometrogram}

In cystometrogram, the contraction interval of the $\mathrm{BOO}$ group $(4.13 \pm 0.15$ minutes) decreased significantly compared with the control group $(10.25 \pm 0.17$ minutes $)(\mathrm{P}<0.05)$. NVCs were observed in the BOO group. In the HM group, the contraction interval was $4.65 \pm 0.24$ minutes, and there was no significant dif- 

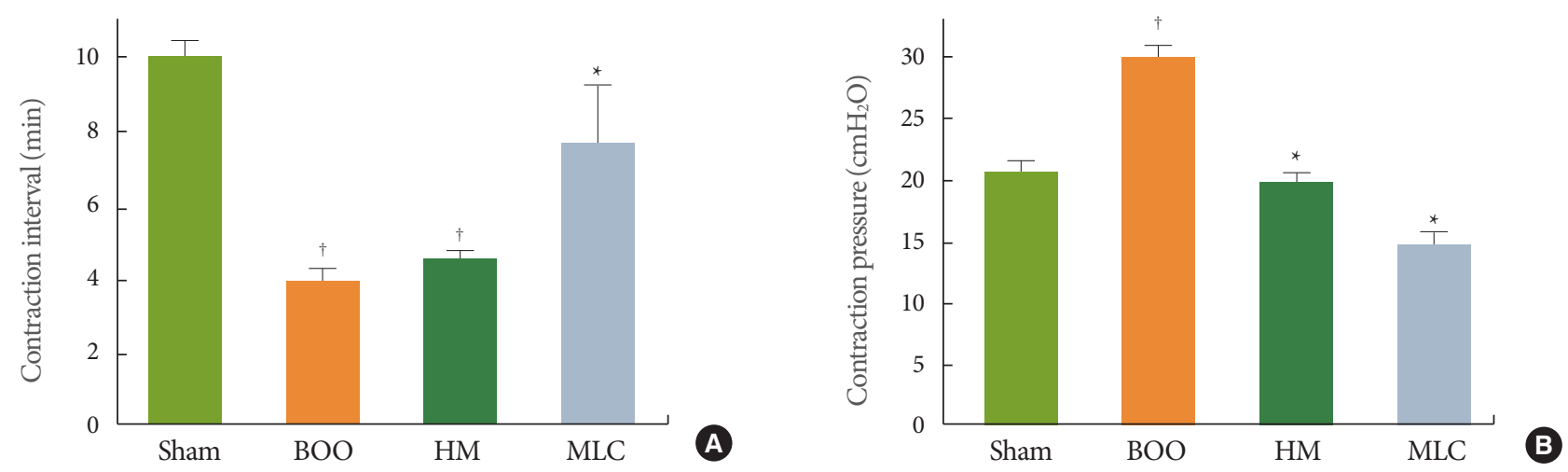

Fig. 1. Changes in the contraction interval (A) and contraction pressure (B) in the control, BOO, HM, and MLC groups. The data are expressed as mean \pm standard error of the mean. ${ }^{\dagger} \mathrm{P}<0.05$ as compared with the sham group. ${ }^{*} \mathrm{P}<0.05$ as compared with the $\mathrm{BOO}$ group. $\mathrm{BOO}$, bladder outlet obstruction; HM, hexamethonium; MLC, methyllycaconitine citrate.
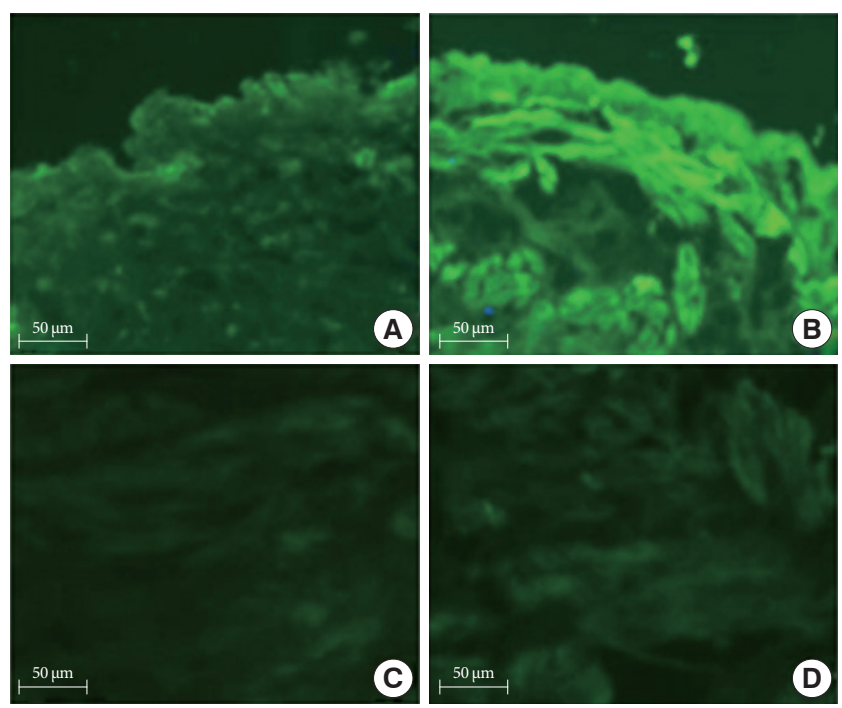

Fig. 2. Immunofluorescence staining of $\alpha 3$ nicotinic acetylcholine receptor of urothelium in the control and $\mathrm{BOO}$ groups. (A) Control urothelium, (B) BOO urothelium, (C) control muscle, and (D) BOO muscle. BOO, bladder outlet obstruction.

ference compared with the BOO group. However, the contraction interval of $7.55 \pm 1.12$ minutes in the MLC group was significantly longer than that in the $\mathrm{BOO}$ group $(\mathrm{P}<0.05)$ (Fig. 1A). In the HM and MLC groups, NVCs were almost lost compared with the BOO group.

The contraction pressure of the BOO group was $30.12 \pm 1.38$ $\mathrm{cmH}_{2} \mathrm{O}$ and significantly higher compared with the control group $\left(20.95 \pm 0.92 \mathrm{cmH}_{2} \mathrm{O}\right)(\mathrm{P}<0.05)$. The contraction pressures of the HM and MLC groups were 19.08 \pm 1.53 and $13.53 \pm 1.02$ $\mathrm{cmH}_{2} \mathrm{O}$, respectively, and significantly lower compared with the
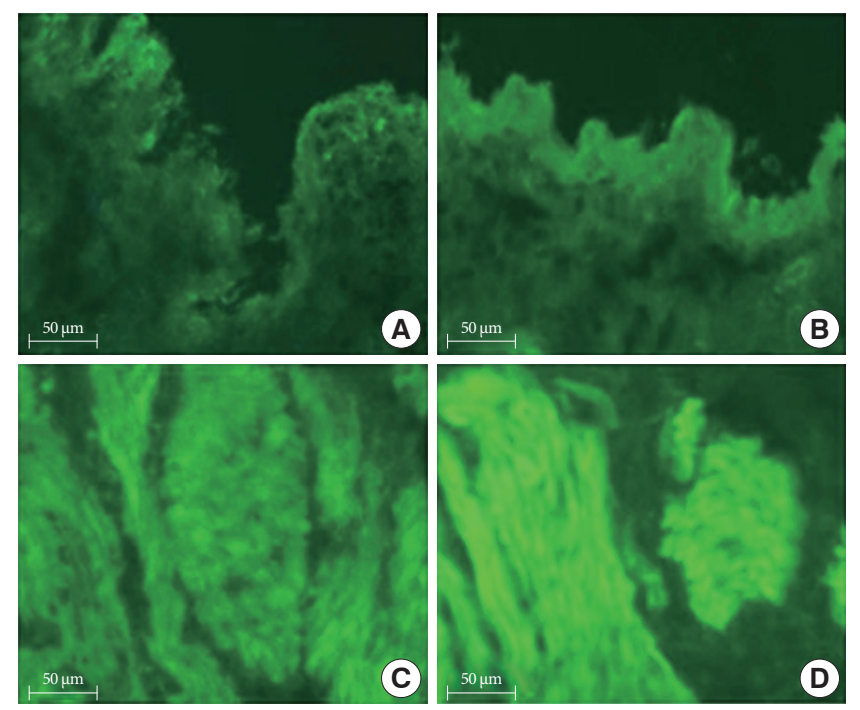

Fig. 3. Immunofluorescence staining of $\alpha 7$ nicotinic acetylcholine receptor of urothelium in the control and $\mathrm{BOO}$ groups. (A) Control urothelium, (B) BOO urothelium, (C) control muscle, and (D) BOO muscle. BOO, bladder outlet obstruction.

BOO group $(\mathrm{P}<0.05)$ (Fig. 1B).

\section{Changes in the Distribution and Expression of a3 and a7 nAChRs by Immunofluorescence}

The $a 3 \mathrm{nAChR}$ expression was observed mainly in the urothelium but had weak expression in the detrusor muscle layer. In the control group, it was observed mainly in the luminal surface of the urothelium. In the BOO group, the expression of $\alpha 3$ $\mathrm{nAChR}$ was significantly increased in the urothelium compared with the control group. However, there was no significant dif- 


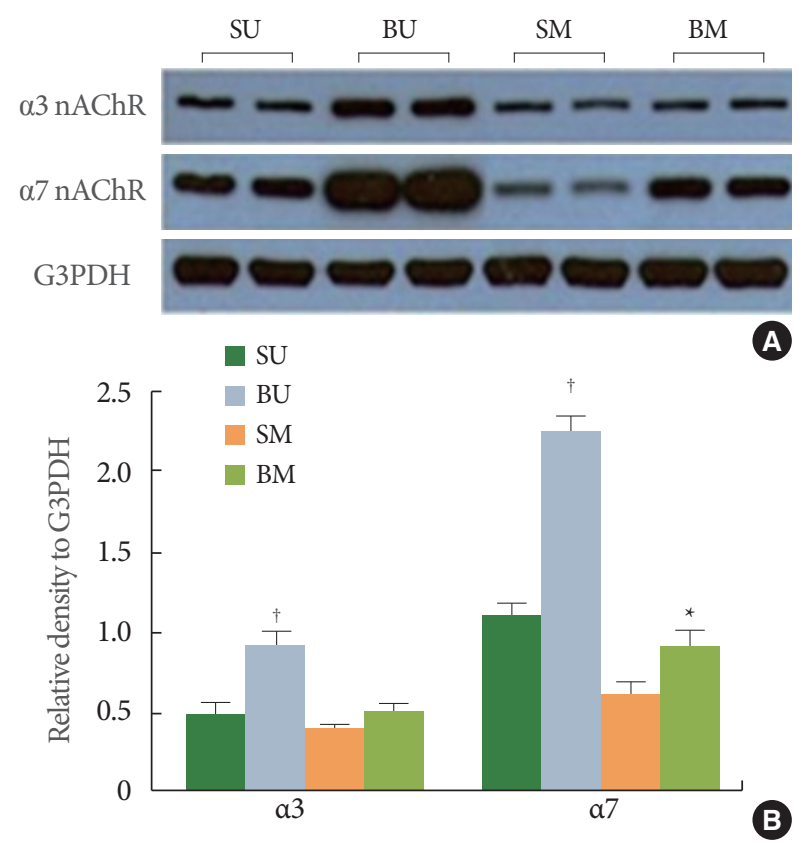

Fig. 4. (A) Protein expression levels of $\alpha 3$ and $\alpha 7$ nicotinic acetylcholine receptor subunits (nAChRs) in the control and $\mathrm{BOO}$ groups. Glyceraldehyde 3-phosphate dehydrogenase (G3PDH) is used as a control gene. (B) Quantitative analysis of the protein levels of $\alpha 3$ and $\alpha 7$ nicotinic acetylcholine receptors in the control and BOO groups, ${ }^{\dagger} \mathrm{P}<0.05$ as compared with the urothelium in the sham group. ${ }^{*} \mathrm{P}<0.05$ as compared with the detrusor muscle in the sham group. BOO, bladder outlet obstruction; SU, sham urothelium; $\mathrm{BU}, \mathrm{BOO}$ urothelium; $\mathrm{SM}$, sham muscle; $\mathrm{BM}$, BOO muscle.

ference from the control group in the detrusor muscle layer (Fig. 2). The $\alpha 7 \mathrm{nAChR}$ expression was observed in both the urothelium and detrusor muscle, unlike $\alpha 3 \mathrm{nAChR}$. In the control group, $\alpha 7 \mathrm{nAChR}$ expression was observed in all layers of the urothelium. In the $\mathrm{BOO}$ group, $\alpha 7 \mathrm{nAChR}$ expression further increased in the urothelium and detrusor muscle compared with the control group (Fig. 3).

\section{Changes in the Expression of $\alpha 3$ and $\alpha 7 \mathrm{nAChRs}$ by Western Blot Analysis}

Expressions of $\alpha 3 \mathrm{nAChR}$ and $\alpha 7 \mathrm{nAChR}$ were confirmed in the mucosa and detrusor muscle. Alpha $3 \mathrm{nAChR}$ expression was significantly increased in the mucosa of the $\mathrm{BOO}$ group compared with the control group $(\mathrm{P}<0.05)$. However, the expression levels in the detrusor muscle were similar for the $\mathrm{BOO}$ and control group. Expression of $\alpha 7 \mathrm{nAChR}$ in the $\mathrm{BOO}$ group significantly increased both in the mucosa and detrusor muscle compared with the control group $(\mathrm{P}<0.05)$ (Fig. 4). These results were in accordance with the immunofluorescence result.

\section{DISCUSSION}

In our study, the distributions of $\alpha 3$ and $\alpha 7 \mathrm{nAChRs}$ were examined in rats in which detrusor overactivity was induced by partial BOO. Although this animal model does not represent detrusor overactivity in humans completely, it has been a mainstay of methods to generate $\mathrm{OAB}$ animal models according to several reports $[17,18]$.

The $\alpha 3 \mathrm{nAChR}$ in the urothelium might play a role in detrusor hyperactivity caused by BOO. So far, little is definitively known about the expression and distribution of $\alpha 3 \mathrm{nAChR}$ in the OAB. Several studies has reported that $\alpha 3 \mathrm{nAChR}$ is involved in bladder contraction. De Biasi et al. [1] reported that mutant mice lacking the a 3 nicotinic subunits developed bladder overdistention, and bladder strips from $\alpha 3$ null mice did not respond to nicotine. Vural et al. [19] reported that HM, a known a 3 nAChR antagonist, inhibited the nicotine-induced increase in the electrical field stimulation-evoked contraction in the rabbit bladder. It can be inferred that the increased levels of $\alpha 3 \mathrm{nAChR}$ and changes in its distribution as well as the structural changes of urothelium caused by BOO might be key factors causing functional changes in the bladder. In addition, definitive information about the expression and distribution of a7 $\mathrm{nAChR}$ in the OAB is lacking. Bschleipfer et al. [20] reported that of the subunits of $n A C h R, \alpha 7$ was more expressed and distributed in the human urothelium without detrusor hyperactivity. Alpha7 nAChR is more likely involved in the regulation of detrusor function. This was confirmed by the findings that the contraction interval was significantly increased, contractile force was decreased, and NVC of the bladder was lost in the cystometrogram in the MLC group compared with the BOO group in our study.

Both immunofluorescence and Western blot analysis confirmed the presence of nAChRs in the urothelium and detrusor muscle. The a $3 \mathrm{nAChR}$ in our study was expressed in the luminal surface of the bladder in the control group. In the $\mathrm{BOO}$ group, the $\alpha 3 \mathrm{nAChR}$ level increased in the urothelium. Alpha7 $\mathrm{nAChR}$ expression was observed in all layers of the urothelium. The expression of $a 7 \mathrm{nAChR}$ in the $\mathrm{BOO}$ group significantly increased compared with the control group. Immunofluorescence and Western blot analysis showed that $\alpha 7 \mathrm{nAChR}$ expression was increased compared with $\alpha 3 \mathrm{nAChR}$ in the bladder 
with detrusor hyperactivity.

Changes in detrusor overactivity were monitored following the intravesicular administration of antagonists against $\alpha 3$ and $\alpha 7 \mathrm{nAChRs}$. There was no significant change in the contraction intervals in the HM group compared with the $\mathrm{BOO}$ group. However, in the HM group, NVC of the bladder was almost lost. The contraction pressure was also significantly decreased compared with the BOO group. These results might relate to the $\alpha 3 \mathrm{nAChR}$ inhibitor, HM and are in agreement with other reports that afferent nerve conduction is activated when $\alpha 3$ $\mathrm{nAChR}$ releases adenosine triphosphate, an excitatory mediator, in response to extrinsic stimulation or extension of the urothelium $[15,21,22]$. The cause of a decrease in the contraction pressure in the HM group may be because the $\mathrm{BOO}$ damaged the urothelium, which increased the permeability of the urothelium to the HM. Hence, HM might have affected the urothelium and detrusor muscle, or the nerve below the urothelium directly. In general, HM mostly affects the $a 3 \mathrm{nAChR}$ present in the urothelium of the normal bladder and has no significant effect on the detrusor muscle. However, in the obstructioninduced detrusor, HM has a direct effect on the detrusor muscle, nerve function of the bladder, as well as the urothelium. Therefore, contraction of the bladder could be suppressed.

In cystometrogram, the contraction interval significantly increased in the MLC group compared with the BOO group. NVC were almost absent and the contraction pressure decreased. According to the other studies, stimulation of $\alpha 7$ nAChR induces the secretion of nitric oxide, an inhibitor of normal urinary bladder function, and thereby, interferes with the neurotransmission of afferent excitation [23-27]. However, those studies differed from our results. Similar to the HM group, MLC may have a direct effect on $a 7 \mathrm{nAChR}$ in the detrusor muscle as well as the urothelium due to the increased permeability of the urothelium caused by the structural changes and damage by BOO. This might lead to the suppression of detrusor hyperreflex. This postulation was in agreement with the report by Beckel et al. [15] that the urothelium is damaged following the intravesicular administration of protamine sulfate in rats with normal bladder function. They also noted the functional changes in the bladder leading to hyperactivity and decreased intervals between contractions following an intravesicular administration of nicotine. In our series, those results were confirmed indirectly following the administration of $\mathrm{nAChR}$ antagonists.

There are several limitations to our study. First, we did not evaluate the residual urine volume in the $\mathrm{BOO}$ animal model. $\alpha 3$ and $\alpha 7 \mathrm{nAChR}$ antagonists may reduce the external sphincter resistance and thus, indirectly reduce the bladder contraction pressure. Therefore, we could not exclude the possibility that the reduction in the contraction pressure is due to the decrease of urethral resistance after the administration of nicotinic receptor antagonists. Second, we used just one dosage each for HM and MLC, but these doses may not produce the maximal effect on the bladder of rats. Further studies are warranted to examine the $\alpha 3$ and $\alpha 7$ nAChRs in the urothelium and detrusor muscles and develop drugs targeting these receptors. Such studies would be helpful for regulating detrusor overactivity caused by $\mathrm{BOO}$ to control symptoms of OAB.

This study confirmed that the $\alpha 3$ and $\alpha 7 \mathrm{nAChRs}$ have roles in inducing detrusor hyperactivity in rats subjected to partial BOO. We also confirmed that the $\alpha 3$ and $\alpha 7 \mathrm{nAChR}$ antagonists regulated this hyperactivity. Further studies are warranted to examine whether detrusor overactivity caused by factors other than obstruction might also be associated with these receptors.

\section{REFERENCES}

1. De Biasi M, Nigro F, Xu W. Nicotinic acetylcholine receptors in the autonomic control of bladder function. Eur J Pharmacol 2000; 393: $137-40$.

2. Wessler I, Kirkpatrick CJ, Racke K. Non-neuronal acetylcholine, a locally acting molecule, widely distributed in biological systems: expression and function in humans. Pharmacol Ther 1998;77:5979.

3. Kawashima K, Fujii T. Basic and clinical aspects of non-neuronal acetylcholine: overview of non-neuronal cholinergic systems and their biological significance. J Pharmacol Sci 2008;106:167-73.

4. Chess-Williams R. Muscarinic receptors of the urinary bladder: detrusor, urothelial and prejunctional. Auton Autacoid Pharmacol 2002;22:133-45.

5. Masuda H, Hayashi Y, Chancellor MB, Kihara K, de Groat WC, de Miguel F, et al. Roles of peripheral and central nicotinic receptors in the micturition reflex in rats. J Urol 2006;176:374-9.

6. Andersson KE. Bladder activation: afferent mechanisms. Urology 2002;59(5 Suppl 1):43-50.

7. Fowler CJ. Bladder afferents and their role in the overactive bladder. Urology 2002;59(5 Suppl 1):37-42.

8. Andersson KE, Yoshida M. Antimuscarinics and the overactive detrusor: which is the main mechanism of action? Eur Urol 2003;43: 
$1-5$.

9. Yoshimura N, Kaiho Y, Miyazato M, Yunoki T, Tai C, Chancellor MB, et al. Therapeutic receptor targets for lower urinary tract dysfunction. Naunyn Schmiedebergs Arch Pharmacol 2008;377:43748.

10. Zarghooni S, Wunsch J, Bodenbenner M, Bruggmann D, Grando SA, Schwantes U, et al. Expression of muscarinic and nicotinic acetylcholine receptors in the mouse urothelium. Life Sci 2007;80:230813.

11. Lindstrom J, Anand R, Gerzanich V, Peng X, Wang F, Wells G. Structure and function of neuronal nicotinic acetylcholine receptors. Prog Brain Res 1996;109:125-37.

12. Mamalaki A, Tzartos SJ. Nicotinic acetylcholine receptor: structure, function and main immunogenic region. Adv Neuroimmunol 1994;4:339-54.

13. Itier V, Bertrand D. Neuronal nicotinic receptors: from protein structure to function. FEBS Lett 2001;504:118-25.

14. Karlin A. Emerging structure of the nicotinic acetylcholine receptors. Nat Rev Neurosci 2002;3:102-14.

15. Beckel JM, Kanai A, Lee SJ, de Groat WC, Birder LA. Expression of functional nicotinic acetylcholine receptors in rat urinary bladder epithelial cells. Am J Physiol Renal Physiol 2006;290:F103-10.

16. Abrams P, Andersson KE. Muscarinic receptor antagonists for overactive bladder. BJU Int 2007;100:987-1006.

17. Parsons BA, Drake MJ. Animal models in overactive bladder research. Handb Exp Pharmacol 2011;(202):15-43.

18. Fry CH, Daneshgari F, Thor K, Drake M, Eccles R, Kanai AJ, et al. Animal models and their use in understanding lower urinary tract dysfunction. Neurourol Urodyn 2010;29:603-8.

19. Vural IM, Ozturk GS, Ercan ZS, Sarioglu Y. Nicotine potentiates the neurogenic contractile response of rabbit bladder tissue via nicotinic acetylcholine receptors: nitric oxide and prostaglandins have no role in this process. Life Sci 2007;80:1123-7.

20. Bschleipfer T, Schukowski K, Weidner W, Grando SA, Schwantes U, Kummer W, et al. Expression and distribution of cholinergic receptors in the human urothelium. Life Sci 2007;80:2303-7.

21. Birder LA, Nealen ML, Kiss S, de Groat WC, Caterina MJ, Wang E, et al. Beta-adrenoceptor agonists stimulate endothelial nitric oxide synthase in rat urinary bladder urothelial cells. J Neurosci 2002;22: 8063-70.

22. Sun Y, Keay S, De Deyne PG, Chai TC. Augmented stretch activated adenosine triphosphate release from bladder uroepithelial cells in patients with interstitial cystitis. J Urol 2001;166:1951-6.

23. Haberberger RV, Henrich M, Lips KS, Kummer W. Nicotinic receptor alpha 7-subunits are coupled to the stimulation of nitric oxide synthase in rat dorsal root ganglion neurons. Histochem Cell Biol 2003;120:173-81.

24. Hawthorn MH, Chapple CR, Cock M, Chess-Williams R. Urothelium-derived inhibitory factor(s) influences on detrusor muscle contractility in vitro. Br J Pharmacol 2000;129:416-9.

25. Templeman L, Chapple CR, Chess-Williams R. Urothelium derived inhibitory factor and cross-talk among receptors in the trigone of the bladder of the pig. J Urol 2002;167(2 Pt 1):742-5.

26. Ozawa H, Chancellor MB, Jung SY, Yokoyama T, Fraser MO, Yu Y, et al. Effect of intravesical nitric oxide therapy on cyclophosphamide-induced cystitis. J Urol 1999;162:2211-6.

27. Li L, Qiu G, Hao P, Song B. Effect of partial bladder outlet obstruction on detrusor compliance, excitability and contractility in rats. Scand J Urol Nephrol 2006;40:293-9. 